\section{Achieving lupus low-disease activity and remission states under belimumab in refractory systemic lupus erythematosus: time and organ involvement matter}

We read with much interest the contribution of Oon et $a l^{1}$ that was published recently in Annals of the Rheumatic Diseases, describing attainment of the lupus low-disease activity state (LLDAS) in a post-hoc analysis of the BLISS trials. At week 52, LLDAS was reached by $12.5 \%$ and $14.4 \%$ of patients treated with belimumab $10 \mathrm{mg} / \mathrm{kg}$ in BLISS-52 and BLISS-76, respectively. The authors conclude that LLDAS is a potential response indicator for future systemic lupus erythematosus (SLE) trials. As opposed to SLE responder index 4 (SRI4), ${ }^{2}$ which represents a change from baseline activity, LLDAS $^{3}$ and remission ${ }^{4}$ are clinical states that should be targeted as they are associated with damage accrual reduction and include the notion of a low prednisone dose. ${ }^{5}$ Yet, these indicators should be further studied before being introduced into clinical trials. In particular, Oon et al's study lacks data on the time needed to achieve LLDAS and the clinical factors influencing its achievement. It is also important to consider the absence of relapse after an improvement of the disease. In this letter, we wish to provide additional data on the time needed, the probability of and the clinical predictors for achieving stable (ie, without subsequent relapse until month 12) LLDAS and remission state under belimumab. We included all patient with SLE treated with belimumab (intravenous $10 \mathrm{mg} /$ $\mathrm{kg}$ on day $0,14,28$ and then every 28 days) in our centre from March 2013 to May 2018 for an active disease despite standard therapy. Follow-up was conducted on a monthly basis during the first 6 months and at months 9 and $12( \pm 1)$ after belimumab initiation. Primary endpoints were defined as reaching stable SRI4, LLDAS or remission state. ${ }^{2-4} 6$ Patients who did not achieve a primary endpoint and stopped belimumab before 12 months were considered as non-responders. Patients who achieved a primary endpoint but stopped belimumab before 12 months were excluded from the final analysis for this endpoint. The secondary endpoint was the time to obtain a stable British Isles Lupus Assessment Group (BILAG) D from a baseline BILAG $\mathrm{A}$ or $\mathrm{B}$ or $\mathrm{C}$ in the musculoskeletal and mucocutaneous systems at month $6( \pm 1)$ and alternatively, at month $12( \pm 1)^{7}$

A total of 50 patients fulfilling the SLICC criteria for SLE were enrolled. ${ }^{8}$ Belimumab indications were persistent arthritis in $44(88 \%)$ cases, active cutaneous lupus in $26(52 \%)$ cases (6 acute cutaneous lupus, 11 subacute cutaneous lupus, 6 discoid rash, 5 chilblains lupus, 7 alopecia, 2 mucosal ulcers and 1 cryoglobulin vasculitis) and serositis in 2 (4\%) cases. Disease activity and previous/ongoing treatments at the beginning of belimumab are displayed in table 1 . Eleven (22\%) patients stopped belimumab before 12 months: 7 treatment failures, 2 adverse events, 1 pregnancy wish and 1 lost to follow-up.

The probability of reaching stable SRI4, LLDAS or remission at month 12 according to Kaplan-Meier estimator were: $81.7 \%, 58.1 \%$ and $37.1 \%$, respectively (figure 1A). The median (25\%-75\% interquartile range) time to meet stable SRI4 was 91 (48-275) days, stable LLDAS 213 (78.5-283.5) days and stable remission 270 (262.5-283.0) days. Survival curves and log-rank test analyses showed that baseline IgG level $<12 \mathrm{~g} / \mathrm{L}$ predicted an increased probability and a shorter timeframe for attaining LLDAS (HR=3.5 (95\% CI 1.4 to 6.9$), \mathrm{p}=0.04$ ) and that the baseline SLICC/American College of Rheumatology (ACR) damage index $\geq 1$ predicted
Table 1 Disease characteristics, previous treatments and ongoing treatments at the beginning of belimumab

\begin{tabular}{|c|c|}
\hline & Patients $(n=50)$ \\
\hline \multicolumn{2}{|l|}{ Disease characteristics at the start of belimumab } \\
\hline SELENA-SLEDAI* score-median (range) & $6(2-14)$ \\
\hline SELENA-SLEDAI score $<4$ & $2(4)$ \\
\hline SELENA-SLEDAI score 4-7 & $31(62)$ \\
\hline SELENA-SLEDAI score $\geq 8$ & $17(34)$ \\
\hline$\geq 1$ BILAG $†$ A or B score & $48(96)$ \\
\hline PGA* — median (range) & $0.6(0.4-1.5)$ \\
\hline Mild/moderate flare* & $41(82)$ \\
\hline Severe flare* & $8(16)$ \\
\hline SDI scoreł—median (range) & $0(0-3)$ \\
\hline Positive Farr assay & $20(40)$ \\
\hline Low C3 & $15(30)$ \\
\hline \multicolumn{2}{|l|}{ Previous treatments } \\
\hline Number of drugs used per patient, median (range) & $4(2-9)$ \\
\hline HCQ & $49(98)$ \\
\hline Chloroquine & $5(10)$ \\
\hline Prednisone & $49(98)$ \\
\hline Methotrexate & $38(76)$ \\
\hline MMF or MPA & $9(18)$ \\
\hline Azathioprine & $10(20)$ \\
\hline Rituximab & $3(6)$ \\
\hline Thalidomide & $5(10)$ \\
\hline Leflunomide & $1(2)$ \\
\hline Colchicine & $3(6)$ \\
\hline Topical tacrolimus & $6(12)$ \\
\hline \multicolumn{2}{|l|}{ Ongoing treatments at the start of belimumab } \\
\hline Hydroxychloroquine & $45(90)$ \\
\hline Patients with (HCQ) $>750 \mathrm{ng} / \mathrm{mL} \S$ & $38 / 45(84)$ \\
\hline Prednisone & $45(90)$ \\
\hline Prednisone dose—mg/day, median (range) & $10(4-50)$ \\
\hline Increase $\|$ of corticosteroids & $16(32)$ \\
\hline Increase of daily prednisone & $13(26)$ \\
\hline Intravenous high-dose methylprednisolone & $9(18)$ \\
\hline Methotrexate & $30(60)$ \\
\hline MMF or MPA & $5(10)$ \\
\hline Azathioprine & $4(8)$ \\
\hline Colchicine & $3(6)$ \\
\hline Thalidomide & $2(4)$ \\
\hline Chloroquine & $2(4)$ \\
\hline Leflunomide & $1(2)$ \\
\hline Topical tacrolimus & $1(2)$ \\
\hline
\end{tabular}

Values are expressed as, $\mathrm{n}(\%)$, unless stated otherwise.

(HCQ), HCQ blood concentration.

*Defined using Petri et al. ${ }^{9}$

tDefined using Isenberg et al. ${ }^{7}$

$\ddagger$ Defined using Gladman et al. ${ }^{10}$

$\S$ Considered to be the therapeutic target.

IDefined as an increase of daily prednisone and/or intravenous high dose (>250 $\mathrm{mg}$ ) methylprednisolone infusion between day 0 (the day of the start of belimumab) and month 1.

BILAG, British Isles Lupus Assessment Group; C3, complement fraction 3; HCQ, hydroxychloroquine; MMF, mycophenolate mofetil; MPA, mycophenolic acid; PGA, physician's global assessment; SDI, SLICC Damage Index; SELENA-SLEDAI, Safety of Estrogens in Lupus Erythematosus: National Assessment version of the Systemic Lupus Erythematosus Disease Activity Index.

a decreased probability and a longer timeframe for attaining SRI4 (HR $=0.4$ (95\%CI 0.2 to 0.4$), p=0.04)$. No significant association was found between the time to achieve SRI4, 
A

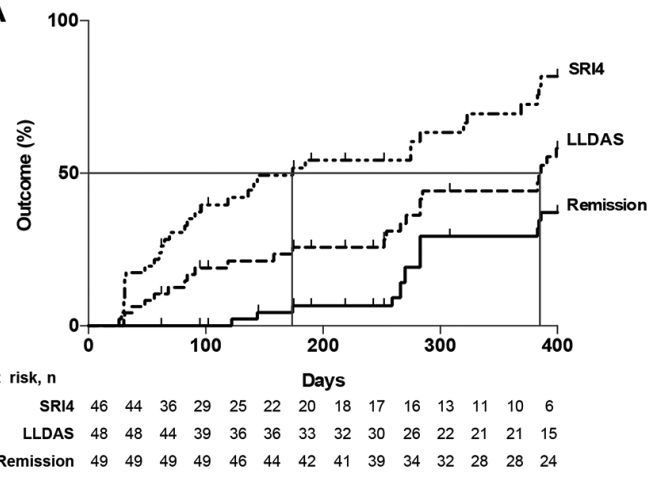

B

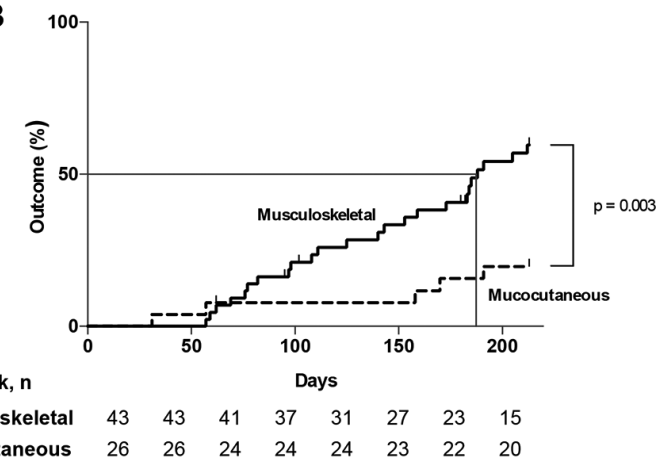

C

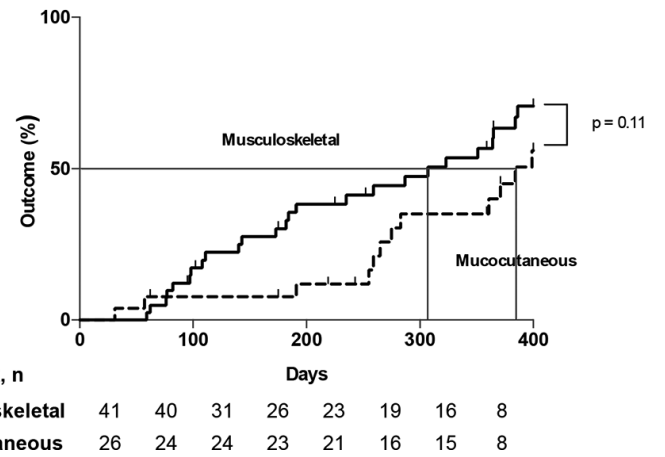

$\begin{array}{lllllllll}\text { Musculoskeletal } & 41 & 40 & 31 & 26 & 23 & 19 & 16 & 8\end{array}$

$\begin{array}{lllllllll}\text { Mucocutaneous } & 26 & 24 & 24 & 23 & 21 & 16 & 15 & 8\end{array}$

Figure 1 SRI4, LLDAS and remission under belimumab treatment. Kaplan-Meier curves. (A) Percentage of patients achieving stable (ie, without subsequent relapse) SRI4, LLDAS and remission. Patients with SLE treated with belimumab were followed for $12( \pm 1)$ months. Each corner in the curve represents a patient who achieved a stable SRI4, LLDAS or remission until $12( \pm 1)$ months. Vertical tick marks along each curve represent patients who did not reach the outcome and had a follow-up shorter than $12( \pm 1)$ months (censored data). Patients who achieved a primary endpoint but who stopped belimumab before $12( \pm 1)$ months were excluded from the final analysis for this endpoint. Numbers of patients at risk for event are reported on a monthly basis for each outcome. The time at which $50 \%$ of the initial population reached the endpoint was 174 days for SRI4 and 386 days for LLDAS. (B and C) Musculoskeletal versus mucocutaneous response at 6 and 12 months. Percentage of patients with a baseline BILAG A, $\mathrm{B}$ or $\mathrm{C}$ in the musculoskeletal system and the mucocutaneous system attaining stable (ie, without subsequent relapse) BILAG D in this system at $6( \pm 1)$ months (B) and $12( \pm 1)$ months (C). Each corner in the curves represents a patient who achieved a stable BILAG D. Data were analysed and censored with the method applied to the primary endpoints. Curves were compared using log-rank tests. BILAG, British Isles Lupus Assessment Group; LLDAS, Lupus Low Disease Activity Score; SELENA-SLEDAI, Safety of Estrogens in Lupus Erythematosus: National Assessment version of the Systemic Lupus Erythematosus Disease Activity Index; SLE, systemic lupus erythematosus; SRI4, SELENA-SLEDAI Responder Index.
LLDAS or remission and the following baseline parameters: Safety of Estrogens in Lupus Erythematosus: National Assessment version of the Systemic Lupus Erythematosus Disease Activity Index (SELENA-SLEDAI) $\geq 6$ or 8 , low C3 serum level, positive Farr assay, daily prednisone dose $\geq 10 \mathrm{mg}$ and concomitant treatment with an immunosuppressant. At month 6 , patients with baseline BILAG A, B or C had an increased probability and a shorter timeframe to meet a stable BILAG $\mathrm{D}$ in the musculoskeletal system compared with the mucocutaneous system $(p=0.003)$. The difference was no longer significant at 12 months $(\mathrm{p}=0.11)$ (figure $1 \mathrm{~B}, \mathrm{C})$.

In conclusion, our study provides evidence that stable LLDAS and remission under belimumab are reached slowly, with a substantial number of patients reaching one or the other after 6 months of treatment and with a faster control of articular activity compared with the cutaneous one. These data suggest that belimumab should probably be maintained for more than 6 months before concluding that there has been an inadequate response, especially in the case of cutaneous lupus. The greater likelihood of obtaining LLDAS in our study compared with the Oon et al's ${ }^{1}$ study was probably related to the higher baseline disease activity seen in the BLISS trials where a SELENA-SLEDAI of at least six was an inclusion criterion.

Nabiha Sbeih, ${ }^{1}$ Alexis Mathian, ${ }^{\oplus 1}$ Marc Pineton de Chambrun, ${ }^{1}$ Raphael Lhote, ${ }^{1}$ Noël Zahr, ${ }^{2}$ Micheline Pha, ${ }^{1}$ Fleur Cohen-Aubart, ${ }^{1}$ Julien Haroche, ${ }^{1}$ Miguel Hié, ${ }^{1}$ Sophie Jouffroy, ${ }^{3}$ Neila Benameur, ${ }^{4}$ Hervé Devilliers, ${ }^{5}$ Zahir Amoura ${ }^{1}$

${ }^{1}$ French National Referral Center for Systemic Lupus Erythematosus, Antiphospholipid Antibody Syndrome and Other Autoimmune Disorders, Service de Médecine Interne 2, Institut E3M, Inserm, Centre d'Immunologie et des Maladies Infectieuses (CIMIParis), Sorbonne Université, AssistancePublique-Hôpitaux de Paris, Groupement Hospitalier Pitié-Salpêtrière, Paris, France

${ }^{2}$ Service De Pharmacologie, Assistance Publique-Hôpitauxde Paris, Groupement Hospitalier Pitié-Salpêtrière, Paris, France

${ }^{3}$ École Nationale Vétérinaire De Toulouse, Toulouse, France

${ }^{4}$ Service De La Pharmacie, Assistance Publique-Hôpitaux de Paris, Groupement Hospitalier Pitié-Salpêtrière, Paris, France

${ }^{5}$ Service De Médecine Interne et Maladies Systémiques (médecine interne 2) et Centre d'Investigation Clinique, Inserm CIC 1432, Centre Hospitalier Universitaire de Dijon, Hôpital François-Mitterrand, Dijon, France

Correspondence to Dr Alexis Mathian, Service de Médecine Interne 2, Hôpital Pitié-Salpêtrière, Paris, France; alexis.mathian@aphp.fr

Contributors NS, AM, RL, HD and ZA contributed to the conception and design of the study; NS, AM, NZ, FCA, JH, MH, MPDC, MP, NB and ZA were involved in the acquisition of data; NS, AM, RL, NZ, FCA, JH, MH, MP, MPDC, SJ, HD and ZA contributed to the analysis and interpretation of data. All authors contributed to drafting and/or revising the manuscript.

Funding The authors have not declared a specific grant for this research from any funding agency in the public, commercial or not-for-profit sectors.

Competing interests $A M$ and ZA have received consulting fees from GSK.

Patient consent for publication Patients were informed that data collected in medical records might be used for research study in accordance to privacy rules.

Ethics approval According to the Public Health French Law, approval from institutional review board is not required for an observational study. Our study involves personal health data and has been authorized by the "Commission nationale de l'informatique et des libertés" (CNIL) (declaration number 2202953).

Provenance and peer review Not commissioned; internally peer reviewed. (c) Author(s) (or their employer(s)) 2019. No commercial re-use. See rights and permissions. Published by BMJ.

NS and AM contributed equally.

NS and AM are joint first authors.

Check for updates 
To cite Sbeih N, Mathian A, Pineton de Chambrun M, et al.

Ann Rheum Dis Epub ahead of print: [please include Day Month Year]. doi:10.1136/ annrheumdis-2019-215732

Received 16 May 2019

Revised 18 June 2019

Accepted 19 June 2019

\section{SLinked}

http://dx.doi.org/10.1136/annrheumdis-2019-215922

Ann Rheum Dis 2019;0:1-3. doi:10.1136/annrheumdis-2019-215732

\section{REFERENCES}

1 Oon S, Huq M, Golder V, et al. Lupus Low Disease Activity State (LLDAS) discriminates responders in the BLISS-52 and BLISS-76 phase III trials of belimumab in systemic lupus erythematosus. Ann Rheum Dis 2019;78:629-33.

2 Furie RA, Petri MA, Wallace DJ, et al. Novel evidence-based systemic lupus erythematosus responder index. Arthritis Rheum 2009;61:1143-51.

3 Franklyn K, Lau CS, Navarra SV, et al. Definition and initial validation of a Lupus Low Disease Activity State (LLDAS). Ann Rheum Dis 2016;75:1615-21.
4 van Vollenhoven R, Voskuyl A, Bertsias G, et al. A framework for remission in SLE: consensus findings from a large international task force on definitions of remission in SLE (DORIS). Ann Rheum Dis 2017;76:554-61.

5 Ugarte-Gil MF, Wojdyla D, Pons-Estel GJ, et al. Remission and Low Disease Activity Status (LDAS) protect lupus patients from damage occurrence: data from a multiethnic, multinational Latin American Lupus Cohort (GLADEL). Ann Rheum Dis 2017;76:2071-4.

6 Wilhelm TR, Magder LS, Petri M. Remission in systemic lupus erythematosus: durable remission is rare. Ann Rheum Dis 2017;76:547-53.

7 Isenberg DA, Rahman A, Allen E, et al. BILAG 2004. Development and initial validation of an updated version of the British Isles Lupus Assessment Group's disease activity index for patients with systemic lupus erythematosus. Rheumatology 2005;44:902-6.

8 Petri M, Orbai A-M, Alarcón GS, et al. Derivation and validation of the Systemic Lupus International Collaborating Clinics classification criteria for systemic lupus erythematosus. Arthritis Rheum 2012;64:2677-86.

9 Petri M, Kim MY, Kalunian KC, et al. Combined oral contraceptives in women with systemic lupus erythematosus. N Engl J Med 2005;353:2550-8.

10 Gladman D, Ginzler E, Goldsmith C, et al. The development and initial validation of the Systemic Lupus International Collaborating Clinics/American College of Rheumatology damage index for systemic lupus erythematosus. Arthritis Rheum 1996:39:363-9. 